\title{
Land- and pool-based intervention in female fibromyalgia patients: A randomized-controlled trial
}

\author{
Agusti Acosta-Gallego ${ }^{1}$ (D) Pedro Jesús Ruiz-Montero² (D), Alfonso Castillo-Rodríguez ${ }^{3}$ (D) \\ 'Department of International University of La Rioja, La Rioja, Spain \\ ${ }^{2}$ Department of Physical Education and Sport, University of Granada, Melilla, Spain \\ ${ }^{3}$ Department of Physical Education and Sport, University of Granada, Granada, Spain
}

Received: November 03, 2017 Accepted: June 12, 2018 Published online: November 07, 2018

\begin{abstract}
Objectives: The aim of this study was to assess the effect of a standard physical rehabilitation intervention (SPRI) program, in pool-based (SPRI-P) and land-based (SPRI-L) environments, applied to female fibromyalgia (FM) patients with mild-to-moderate symptoms during a period of 20 weeks.

Patients and methods: Between September 2016 and September 2017, a total of 73 female FM patients (mean age $48.2 \pm 6.8$ years; range, 30 to 59 years) who suffered from FM were included in this study on a voluntary basis. The SPRI program was applied to the patients for 20 weeks. The severity of FM was assessed using the Fibromyalgia Impact Questionnaire. The dependent variables including selfperceived pain, perceived fatigue, aerobic capacity, depressive symptoms, and the overall impact of FM were evaluated.

Results: Self-perceived pain, perceived fatigue, overall impact of FM $(\mathrm{p}<0.05)$ and depressive symptoms $(\mathrm{p}<0.001)$ of the participants in the SPRI-P program decreased in the post-test values, compared to the pre-test values. Using the SPRI-L program, a significant improvement in the aerobic capacity (maximum consumption of relative oxygen and distance in meters), overall impact of $F M(p<0.05)$, and depressive symptoms $(\mathrm{p}<0.01)$ was observed.

Conclusion: Our study results indicated that the SPRI-P program yielded improvements in patients with FM in self-perceived pain, perceived fatigue, depressive symptoms, and overall impact, as well as improving the aerobic capacity. In addition, in the SPRI-L program, the participants increased their capacities, self-perceived pain, and overall impact of FM.
\end{abstract}

Keywords: Female; fibromyalgia; pain; physical activity; interventional; program.

Fibromyalgia (FM) is a process of chronic and generalized pain, without a recognizable cause, with a duration of more than three months and increased tenderness to palpation with a generalized pain, defined as pain in at least four of five regions (left upper, right upper, left lower, right lower, axial). ${ }^{[1]}$ In addition, FM is characterized by a decrease in pain threshold and symptoms such as sleep disorders, fatigue, rigidity, altered emotional states, depression, anxiety, cognitive dysfunction, irritable bowel syndrome, migraine headaches, and paresthesia (tingling). ${ }^{[2]}$

The prevalence of FM worldwide has been reported as ranging between 0.5 and $5 \%^{[3,4]}$ with higher frequencies among individuals with a family history (i.e., genetic and environmental influence). ${ }^{[5]}$ In Spain, this prevalence is $2.4 \%$, affecting a significantly higher proportion of women $(4.2 \%)$ than men $(0.2 \%)$ and adults aged between 40 and 49 years. ${ }^{[6]}$

Fibromyalgia is associated with decreased physical function in patients $s^{[7]}$ and, in many cases, altered psychological function such as depression, anxiety or memory disorders. ${ }^{[8]}$ Also, exposure to psychosocial and environmental stressors, along with alterations in the autonomic nervous system and in the neuroendocrine responses, contributes to dysfunctions in pain perception. ${ }^{[9]}$ Jones et al. ${ }^{[7]}$ reported difficulties among female FM patients in performing the tasks associated with physical independence. However, 
a large number of FM interventional programs has been used as effective treatment modalities. ${ }^{[10]}$ Health status, depressed mood or average pain intensity show significant changes with interventional programs through multimodal programs. ${ }^{[1]}$ Regular physical exercise improves the functional capacity in FM patients. In addition, interventional programs with physical exercises are shown to help with the management of the disease. ${ }^{[12]}$ Most of the studies on physical exercise and interventional programs in FM patients have focused on the aerobic capacity and/or muscular strength. ${ }^{[13]}$ Aerobic exercise produces positive effects on FM patients, ${ }^{[14]}$ while the strength exercise not always influence positively on FM symptoms. ${ }^{[13]}$

Furthermore, FM has a profound impact on the patient life, causing a decline in the quality of life, which negatively affects social and professional functions. ${ }^{[15]}$ Many authors recognize the need of more research on this subject to gain a better understanding of the true physiopathology of FM. ${ }^{[5,16]}$ However, the constant pressure of the symptoms, typical for FM, changes the patient life habits as the severity and unpredictability of these symptoms make it difficult to adequately carry out work tasks or leisure time activities.

In the present study, we aimed to assess the effects (self-perceived pain, perceived fatigue, aerobic capacity, depressive symptoms and the overall impact of FM) of a standard physical rehabilitation intervention (SPRI) program, in pool-based (SPRI-P) and land-based (SPRI-L) environments, applied to female FM patients with mild-to-moderate symptoms of FM.

\section{PATIENTS AND METHODS}

\section{Participants}

In this randomized-controlled trial, a total of 181 FM patients were willing to participate on a voluntary basis between September 2016 and September 2017. After explaining the procedure, objectives, and the possible risks and benefits, 90 patients signed an informed consent form. Seventeen patients who did not fulfill the required ratio of attendance stipulated at $90 \%$ or else left the program due to personal reasons or a worsening physical state were excluded. Finally, a total of 73 female FM patients (mean age 48.2 \pm 6.8 years; range, 30 to 59 years) were included in this study. Figure 1 shows an overview of the patient in the study based on the randomized-control trial flow diagram.

The inclusion criteria were as follows: female sex, aged 30 to 59 years; having an accredited medical certificate with an established diagnosis of FM according to the American College of Rheumatology; ${ }^{[1]}$

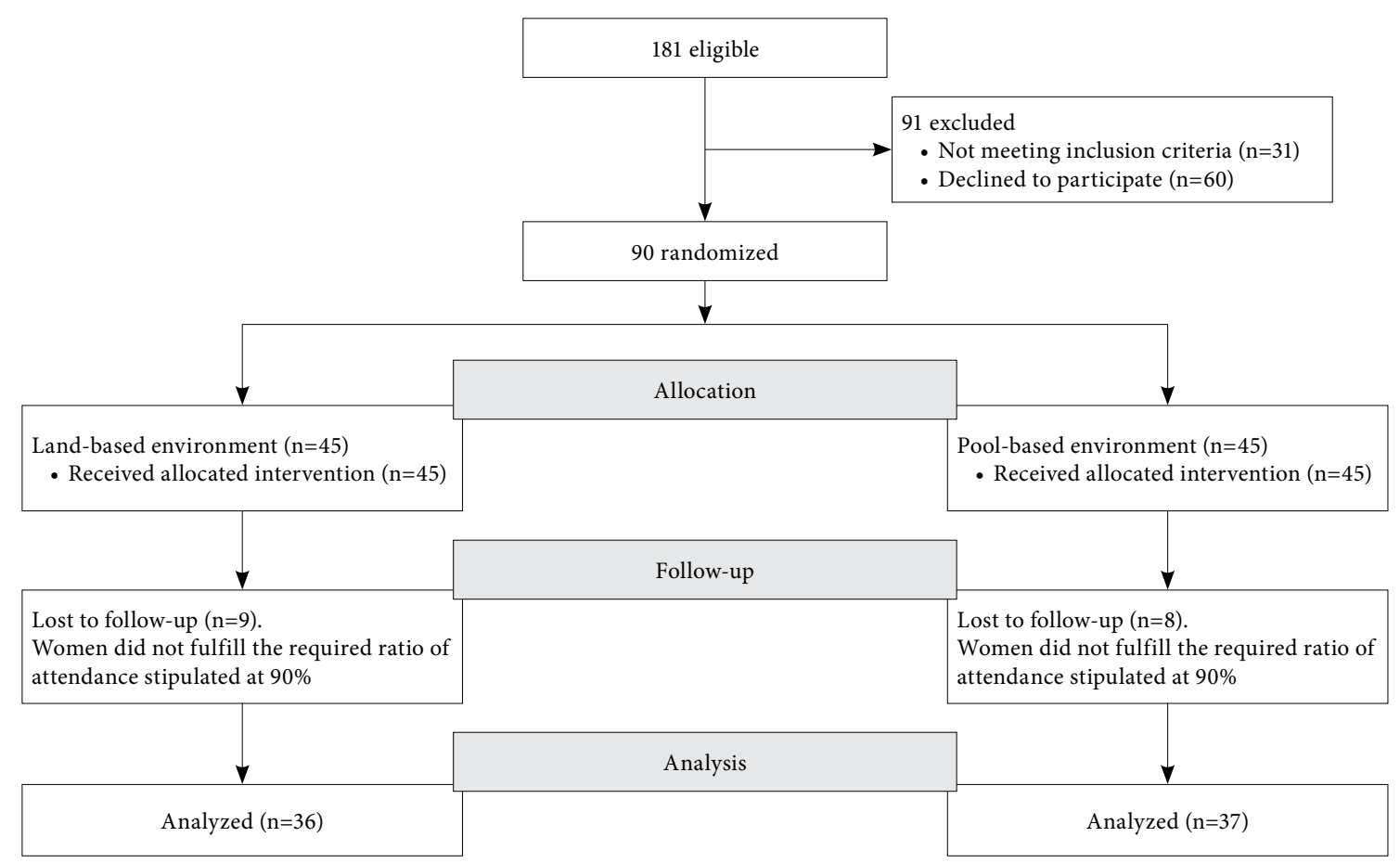

Figure 1. Flow diagram for randomized control study. 
experience a mild-to-moderate degree of impairment due to FM based on the Fibromyalgia Impact Questionnaire (FIQ) <70); ${ }^{[17]}$ and being capable of walking without aid. The exclusion criteria were as follows: having an unstable cardiovascular pathology or any other medical condition to impede physical exercise; having a history of fractures of the upper or lower members during the last three months; having a neuromuscular disease or ingestion of drugs which can affect the neuromuscular function.

\section{Instruments}

Semi-structured interview was used to collect socio-demographic, clinical and physical data.

Heart Rate Monitor (Polar RC3, Polar Pharma Inc., Hollola, Finland) was used to measure the heart rate (HR).

Scale and height rod (Tanita SC-330, Tanita Corporation, Tokyo, Japan) were used to measure weight and height of patient from which we calculated Body Mass Index (BMI). ${ }^{[18]}$

Visual Analog Scale (VAS) was used to measure the intensity of self-perceived pain by the patient. ${ }^{[19]}$ This scale is $10 \mathrm{~cm}$ in length. One end corresponds to no pain (0), the other to maximum pain (10). Slight pain is considered to be VAS $<3.3$, moderate pain VAS 3.3-6.6, severe pain VAS 6.7-9.9, and unbearable pain VAS 10.

Six Minute Walk Test (6MWT) was used to measure the functional respiratory and aerobic capacity. It is the instrument best tolerated by patients, ${ }^{[20,21]}$ recommended by the American College of Sports Medicine (ACSM): ${ }^{[22]}$ VO2Max. $=0.2 \mathrm{~mL} / \mathrm{kg} / \mathrm{min}$ (distance) $+3.5 \mathrm{~mL} / \mathrm{kg} / \mathrm{min}$ (time).

Hamilton Depression Scale (HAM-D) was used to measure depressive symptoms. ${ }^{[23]}$ It is composed of 17 items. It mainly focuses on the somatic and behavioral aspects of depression, but also measures vegetative, cognitive and anxiety symptoms. The overall score is calculated by adding the scores of each item, and ranges between 0 and 54: very severe $>23$, severe 19-22, moderate 14-18, mild 8-13, and normal $0-7$. Internal consistency showed an alpha coefficient of 0.89 for the total items of HAM-D.

The FIQ was used to measure the overall impact of FM. The reliability and validity of this scale have been performed by Rivera. ${ }^{[17]}$ Internal consistency showed an alpha coefficient of 0.82 for the total items of FIQ. It is composed of 10 rating subscales scored between 0 and 10: functional impairment, overall well being (the number of days the patient felt well during the previous week), missing working days (the days of work absence), and seven subscales using a $10-\mathrm{cm}$-long VAS, on which the patient rates work difficulty, pain, fatigue, morning tiredness, stiffness, anxiety, and depression. The total score ranges from 0 to 100 , being a higher values indicate greater impact of the disorder. ${ }^{[17]}$

\section{Procedure}

This randomized-controlled trial protocol was approved by the Ethics Committee of the International University of Catalunya (Spain) and registered by ISRCTN Registry (registration number: ISRCTN17382522; http://doi.org/10.1186/ ISRCTN17382522). The study was conducted in accordance with the principles of the Declaration of Helsinki. A written informed consent was obtained from each participant.

Initially, an interview was applied to all patients, followed by questionnaires and a physical test. All physical-aerobic exercises were conducted on the same day. The random allocation sequence was generated by the investigators. The participants were assigned to either the treatment or control group using the sealed envelope method.

The characteristics of the SPRI program (in the pool-based and land-based environments) were created by physiotherapists and professionals in Sport Science and Physical Education. The recommendations of the ACSM for exercise testing and prescription were considered. The program duration was 20 weeks with two 50-min sessions each week.

The structure of the SPRI program was divided into four stages: (i) warm-up, (ii) main aerobic exercise, (iii) soft stretching, and (iv) relaxation. The content of each stage was similar in both environments, with the activities and exercises adapted to the particular environment. The first two sessions were not similar as the pool-based program started out with an adaptation phase where the patient became familiar with being in water and became conscious of her posture in this medium.

In the warm-up stage, each session began with a 10-min warm-up which consisted of activities based primarily on walking (slower or faster). The participants were asked to walk sideways or backwards, to change their pace, or to pass a ball between them while walking, etc. The main exercise was the most important part of the SPRI program, and consisted of a mix of activities based on aerobic exercise, proprioception, balance, and breathing. The principal of progressive load increase 
was taken into consideration, and the patients never attained high intensity workloads. This period lasted approximately $25 \mathrm{~min}$. Stretching and relaxation was carried out in the end of the exercise period and performed for $10 \mathrm{~min}$, focusing on the muscles most used.

\section{Statistical analysis}

Statistical analysis was performed using the IBM SPSS for Windows version 21.0 software (IBM Corp., Armonk, NY, USA). Normality of distribution was analyzed using the Kolmogorov-Smirnov test, showing a non-normal distribution of variables. Descriptive data were expressed in mean and standard deviation (SD). Coefficient Cronbach's alpha for all composite variables were lower than the acceptance reliability value of $0.80(\alpha=0.66)$. The comparison of means with independent variables using the Mann-Whitney U test and the test for repeated measures using the Wilcoxon test (paired samples) were carried out. The dependent variables in this study were as follows: self-perceived pain, perceived fatigue, aerobic capacity, depressive symptoms, and overall impact of FM. The independent variables included SPIR in the pool-based environment and SPIR in the land-based environment. A specific statistical analysis was performed to calculate the sample size minimum needed for the analysis. Considering a statistical power of $80 \%$, a type I error or alpha of 0.05 and effect size of 0.8 of all dependent variables, a minimum sample size of 52 subjects was needed. The sample size was calculated according to the recommendations reported in the study of HaijanTelaki. ${ }^{[24]}$ The magnitude of the differences between pre- and post-tests of active and control groups were calculated using the effect size. ${ }^{[25]}$ This effect size was calculated using the formula $d=$ (Mpost - Mpre)/ SDpooled, where Mpost is the mean for the post-test and Mpre is the mean for the pre-test, and SDpooled is the pooled SD of the measurements. It has been recommended that effect size should be corrected for the magnitude of the sample size of each study. ${ }^{[26]}$ The effect size can be interpreted as small $(0.2<\mathrm{d}<0.5)$, medium $(0.5<\mathrm{d}<0.8)$ or large $(0.8<\mathrm{d})$. A $p$ value of less than 0.05 was considered statistically significant.

\section{RESULTS}

Of a total of 73 patients with FM, the mean age was $48.2 \pm 6.8$ (range, 30 to 59) years and the mean BMI was $27.67 \pm 5.27 \mathrm{~kg} / \mathrm{m}^{2}$. Table 1 shows the sociodemographic and clinical characteristics of the patients included in the SPRI program. There was no significant difference in the demographic and clinical characteristics among the patients.

With regard to the dependent variables of the study, several differences were found between the preand post- tests of the SPRI-P and the SPRI-L groups (Table 2). For self-perceived pain, in the SPRI-P, there was a reduction in post-test values compared to pretest $(p<0.01)$. In the SPRI-L the scores were similar in the pre-test and the post-test. For perceived fatigue, there was also a reduction in post-test scores compared to pre-test $(\mathrm{p}<0.05)$, although no similar reduction was found in the SPRI-L. For the aerobic capacity, VO2, as well as distance achieved were measured. For both variables there was no improvement in the SPRI-P ( $>0.05$ ), while there was an increase in post-test scores in the SPRI-L for VO2, as well as for meters run $(\mathrm{p}<0.05)$. As far as depressive symptoms, there was a considerable improvement found in both programs (SPRI-P and SPRI-L) with reduced symptoms $\mathrm{p}<0.001$ ). Finally, for overall impact of FM there was a reduction in the SPRI-P $(\mathrm{p}<0.05)$; however, in the SPRI-L the FIQ scores were higher, indicating an increase in the overall impact $(p<0.01)$. According to the effect size, self-perceived pain and depressive symptoms in the SPRI-P were interpreted as medium, whereas the depressive symptoms in the SPRI-L were interpreted as medium.

Table 1. Demographic and anthropometric characteristics of participants $(n=73)$

\begin{tabular}{|c|c|c|c|c|c|c|c|}
\hline \multirow[b]{2}{*}{ Variable } & \multicolumn{3}{|c|}{ SPRI-P $(n=37)$} & \multicolumn{3}{|c|}{ SPRI-L (n=36) } & \multirow[b]{2}{*}{$p$} \\
\hline & Mean \pm SD & Mean & Min-Max & Mean \pm SD & Mean & Min-Max & \\
\hline Age (years) & $58.05 \pm 6.58$ & 58 & $55-70$ & $58.25 \pm 7.12$ & 58 & $55-70$ & 0.903 \\
\hline Weight (kg) & $70.73 \pm 15.4$ & 67.3 & $59.6-89.1$ & $66.09 \pm 10.5$ & 65.1 & $58.1-83.9$ & 0.137 \\
\hline Height $(\mathrm{cm})$ & $158.0 \pm 5.39$ & 160 & $151-169$ & $156.7 \pm 5.43$ & 159 & $150-169$ & 0.337 \\
\hline BMI $\left(\mathrm{kgm}^{-2}\right)$ & $28.28 \pm 5.70$ & 27.2 & $23.4-35.1$ & $27.03 \pm 4.79$ & 26.8 & $23.9-36.3$ & 0.312 \\
\hline
\end{tabular}

SPRI-P: Standard Physical Rehabilitation Intervention program in the pool-based; SPRI-L: Standard Physical Rehabilitation Intervention program in the land-based; SD: Standard deviation; Min: Minimum; Max: Maximum; BMI: Body Mass Index; Mann-Whitney U test. 


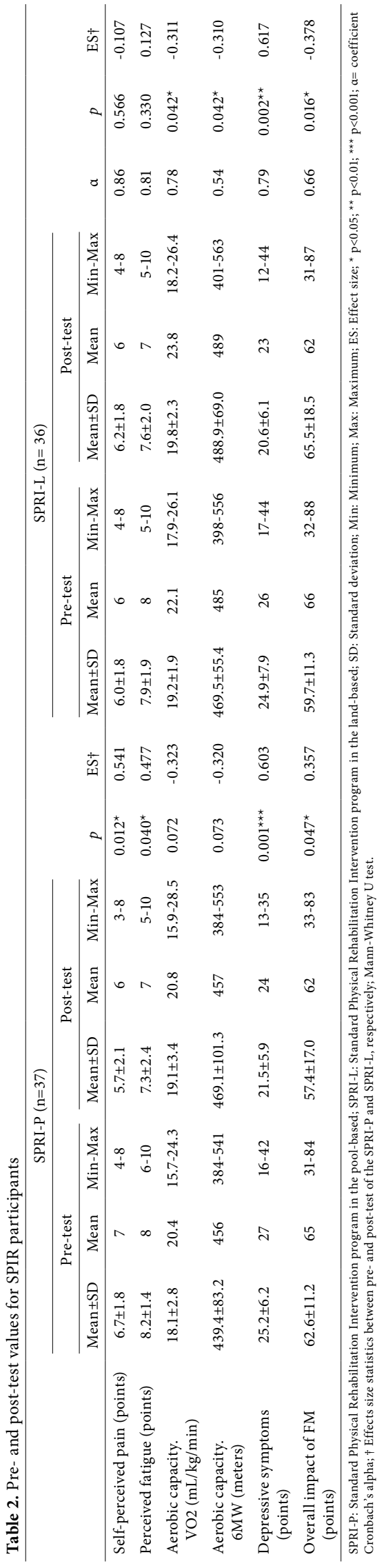

\section{DISCUSSION}

In the present study, we evaluated the effects (i.e., self-perceived pain, perceived fatigue, aerobic capacity, depressive symptoms and the overall impact of FM) of a SPRI in pool-based and land-based environments in women with FM.

A reduction in self-perceived pain was observed in post-test results consistent with other studies carried out in the pool-based environment with female patients suffering from $\mathrm{FM},{ }^{[27,28]}$ which confirm that physical exercise programs conducted in this environment are beneficial for self-perceived pain in patients with similar characteristics. Nevertheless, in the SPRI-L group we observed a slight increase in the self-perceived pain ( $p>0.05)$. This is also consistent with similar studies conducted in a traditional landbased environment using aerobic exercise, strength building, and stretching in the same manner as in our study. ${ }^{[27,29]}$ Cazzola et al. ${ }^{[30]}$ showed that, when designing a physical exercise program for patients with FM, it was critical that the determination of workload does not aggravate self-perceived pain after exercise. In designing of the present physical-combined program, selected exercises were from low to moderate intensity. This proved to be adequate for the SPRI-P group, but not for the SPRI-L group.

The pool-based environment, with its characteristics of buoyancy (which reduces the effects of gravity), resistance of water (which reduces impact on joints, muscles and tendons), and the relaxing effect water has on muscles, prevent possible post-exercise pain and foster the benefits produced by the training session. ${ }^{[3]}$ Along these lines, several studies have assessed the effects on pain for patients with FM in both environments, showing greater benefits attained in the pool-based group. ${ }^{[32]}$

This difference between the groups was also observed with perceived fatigue, namely, the poolbased group showed an improvement in scores. These results correspond with a study of female participants with FM in a 12-week pool-based aerobic exercise program. ${ }^{[33]}$ However, despite the fact that the mildto-moderate intensity of the exercises was adequate in both environments, no improvement was found in the SPRI-L group, consistent with a previous study. ${ }^{[14]}$

In the present study, we found an increase in aerobic capacity in both groups (pool-based and land-based environments), although in the pool-based group these results were not significant. Previous studies have also shown similar significant improvements 
in the aerobic capacity. ${ }^{[29,34]}$ Although the period of intervention was shorter (five months) for our program, compared to the study of Tomas-Carus et al. ${ }^{[34]}$ (eight months), we still found an improvement in the aerobic capacity. The benefits obtained in the aerobic capacity in the land-based environment may be related to physiological responses, given that the moderate exertion exercised in this environment presupposes greater oxygen consumption than for the pool-based environment. ${ }^{[34]}$ Moreover, some studies have demonstrated an improvement in the aerobic capacity. ${ }^{[29,31,33]}$ Based on these findings, it is possible to conclude that educational programs based on strategies for coping with FM symptoms should be implemented.

In our study, the results obtained for depressive symptoms showed an improvement in patients in both SPRI-P and SPRI-L groups, similar to previous findings reported in the literature. ${ }^{[32,33,35]}$ Patients with FM tend to like working in the pool-based environment. This results in more active participation, which encourages a disconnection from daily problems and explains the reduction in depressive symptoms found in patients with FM. ${ }^{[36]}$

Finally, the FIQ is a widely used tool in studies of the effect of physical exercise programs on patients with FM. In our study, a reduction in the FIQ scores in the SPRI-P group was observed, consistent with the findings of López-Rodriguez et al. ${ }^{[37]}$ whose programs of intervention were based on Biodanza and Tai Chi respectively. Previously, similar results were obtained with programs in the pool-based environment. ${ }^{[33]} \mathrm{On}$ the other hand, the increase seen in the FIQ scores in the SPRI-L group is consistent with a study of a 15-week program of sessions consisting of Nordic walking and stretching, ${ }^{[21]}$ although other studies have shown a reduction in the FIQ. ${ }^{[37,38]}$ These differences may be due to sampling differences. Specifically, the patients in our study showed high levels of perceived fatigue, a VO2max slightly below values indicating physical inactivity, and a BMI corresponding to overweight. In this context, the patients who participated in the SPRI-L group had an extra handicap. As the landbased environment implies a greater impact on the performance of physical exercise, it together with work overload due to being overweight and having a low aerobic capacity, might have caused an increase in the perceived pain. This, in turn, would result in less active participation which could explain the negative results found in our study in terms of the overall impact of FM on the SPRI-L group.
Nonetheless, there are some limitations to this study. First, the study included only female patients and, therefore, it is difficult to generalize the findings to the overall population. Second, our participants were volunteers, which could have affected the representativeness of the study sample. Therefore, this might have induced possible differences across the two groups.

In conclusion, our study results demonstrate that the SPRI program produces improvements in clinical manifestations in patients with FM with reduced physical symptoms (self-perceived pain, perceived fatigue) and increased functional capacity (aerobic capacity), as well as decreased associated symptoms (depressive). However, it must be taken into account that these improvements may vary according to the environment in which the program is performed (pool-based or land-based). Based on our study findings, we conclude that SPRI-P program is more effective, yielding a reduction in the overall impact of FM on these patients.

\section{Declaration of conflicting interests}

The authors declared no conflicts of interest with respect to the authorship and/or publication of this article.

\section{Funding}

The authors received no financial support for the research and/or authorship of this article.

\section{REFERENCES}

1. Wolfe F, Smythe HA, Yunus MB, Bennett RM, Bombardier C, Goldenberg DL, et al. The American College of Rheumatology 1990 Criteria for the Classification of Fibromyalgia. Report of the Multicenter Criteria Committee. Arthritis Rheum 1990;33:160-72.

2. Arnold LM, Clauw DJ, McCarberg BH. Improving the recognition and diagnosis of fibromyalgia. Mayo Clin Proc 2011;86:457-64.

3. Neumann L, Buskila D. Epidemiology of fibromyalgia. Curr Pain Headache Rep 2003;7:362-8.

4. White KP, Harth M. Classification, epidemiology, and natural history of fibromyalgia. Curr Pain Headache Rep 2001;5:320-9.

5. Chakrabarty S, Zoorob R. Fibromyalgia. Am Fam Physician 2007;76:247-54.

6. Mas AJ, Carmona L, Valverde M, Ribas B. Prevalence and impact of fibromyalgia on function and quality of life in individuals from the general population: results from a nationwide study in Spain. Clin Exp Rheumatol 2008;26:519-26.

7. Jones J, Rutledge DN, Jones KD, Matallana L, Rooks DS. Self-assessed physical function levels of women with fibromyalgia: a national survey. Womens Health Issues 2008;18:406-12. 
8. Smith HS, Harris R, Clauw D. Fibromyalgia: an afferent processing disorder leading to a complex pain generalized syndrome. Pain Physician 2011;14:217-45.

9. Harkness EF, Macfarlane GJ, Nahit ES, Silman AJ, McBeth J. Risk factors for new-onset low back pain amongst cohorts of newly employed workers. Rheumatology (Oxford) 2003;42:959-68.

10. Van Abbema R, Van Wilgen CP, Van Der Schans CP, Van Ittersum MW. Patients with more severe symptoms benefit the most from an intensive multimodal programme in patients with fibromyalgia. Disabil Rehabil 2011;33:743-50.

11. Lemstra M, Olszynski WP. The effectiveness of multidisciplinary rehabilitation in the treatment of fibromyalgia: a randomized controlled trial. Clin J Pain 2005;21:166-74.

12. Genc A, Tur BS, Aytur YK, Oztuna D, Erdogan MF. Does aerobic exercise affect the hypothalamic-pituitary-adrenal hormonal response in patients with fibromyalgia síndrome? J Phys Ther Sci 2015;27:2225-31.

13. Akyol Y, Ulus Y, Tander B, Bilgici A, Kuru Ö. Muscle strength, fatigue, functional capacity, and propioceptive acuity in patients with fibromyalgia. Turk J Phys Med Rehab 2013;59:292-8.

14. Vural M, Berkol TD, Erdogdu Z, Pekedis K, Kuçukserat B, Aksoy C. Evaluation of the effectiveness of an aerobic exercise program and the personality characteristics of patients with fibromyalgia syndrome: a pilot study. J Phys Ther Sci 2014;26:1561-5.

15. Cunningham MM, Jillings C. Individuals' descriptions of living with fibromyalgia. Clin Nurs Res 2006;15:258-73.

16. Schmidt-Wilcke T, Clauw DJ. Fibromyalgia: from pathophysiology to therapy. Nat Rev Rheumatol 2011;7:518-27.

17. Rivera J, González T. The Fibromyalgia Impact Questionnaire: a validated Spanish version to assess the health status in women with fibromyalgia. Clin Exp Rheumatol 2004;22:554-60.

18. Arranz L, Canela MA, Rafecas M. Relationship between body mass index, fat mass and lean mass with SF-36 quality of life scores in a group of fibromyalgia patients. Rheumatol Int 2012;32:3605-11.

19. Boonstra AM, Schiphorst Preuper HR, Reneman MF, Posthumus JB, Stewart RE. Reliability and validity of the visual analogue scale for disability in patients with chronic musculoskeletal pain. Int J Rehabil Res 2008;31:165-9.

20. Revill SM, Noor MZ, Butcher G, Ward MJ. The endurance shuttle walk test: an alternative to the six-minute walk test for the assessment of ambulatory oxygen. Chron Respir Dis 2010;7:239-45.

21. Mannerkorpi K, Nordeman L, Cider A, Jonsson G. Does moderate-to-high intensity Nordic walking improve functional capacity and pain in fibromyalgia? A prospective randomized controlled trial. Arthritis Res Ther 2010;12:189.

22. American College of Sports Medicine's (ACSM) Guidelines for Exercise Testing and Prescription. 10th ed. Philadelphia: Lippincott; 2017.
23. Hamilton M. Development of a rating scale for primary depressive illness. Br J Soc Clin Psychol 1967;6:278-96.

24. Hajian-Tilaki K. Sample size estimation in epidemiologic studies. Caspian J Intern Med 2011;2:289-98.

25. Cohen J. A power primer. Psychol Bull 1992;112:155-9.

26. Hedges LV, Olkin I. Statistical Methods for Meta-analysis. New York, NY: Academic Press; 1985.

27. Newcomb LW, Koltyn KF, Morgan WP, Cook DB. Influence of preferred versus prescribed exercise on pain in fibromyalgia. Med Sci Sports Exerc 2011;43:1106-13.

28. Tomas-Carus P, Häkkinen A, Gusi N, Leal A, Häkkinen $\mathrm{K}$, Ortega-Alonso A. Aquatic training and detraining on fitness and quality of life in fibromyalgia. Med Sci Sports Exerc 2007;39:1044-50.

29. Hooten WM, Qu W, Townsend CO, Judd JW. Effects of strength vs aerobic exercise on pain severity in adults with fibromyalgia: a randomized equivalence trial. Pain 2012;153:915-23.

30. Cazzola M, Atzeni F, Salaffi F, Stisi S, Cassisi G, SarziPuttini P. Which kind of exercise is best in fibromyalgia therapeutic programmes? A practical review. Clin Exp Rheumatol 2010;28:117-24.

31. Mannerkorpi K, Hernelid C. Leisure Time Physical Activity Instrument and Physical Activity at Home and Work Instrument. Development, face validity, construct validity and test-retest reliability for subjects with fibromyalgia. Disabil Rehabil 2005;27:695-701.

32. Evcik D, Yigit I, Pusak H, Kavuncu V. Effectiveness of aquatic therapy in the treatment of fibromyalgia syndrome: a randomized controlled open study. Rheumatol Int 2008;28:885-90.

33. de Andrade SC, de Carvalho RF, Soares AS, de Abreu Freitas RP, de Medeiros Guerra LM, Vilar MJ. Thalassotherapy for fibromyalgia: a randomized controlled trial comparing aquatic exercises in sea water and water pool. Rheumatol Int 2008;29:147-52.

34. Tomas-Carus P, Gusi N, Häkkinen A, Häkkinen K, Leal A, Ortega-Alonso A. Eight months of physical training in warm water improves physical and mental health in women with fibromyalgia: a randomized controlled trial. J Rehabil Med 2008;40:248-52.

35. Gusi N, Tomas-Carus P. Cost-utility of an 8-month aquatic training for women with fibromyalgia: a randomized controlled trial. Arthritis Res Ther 2008;10:24.

36. Sañudo B, Carrasco L, de Hoyo M, McVeigh JG. Effects of exercise training and detraining in patients with fibromyalgia syndrome: a 3-yr longitudinal study. Am J Phys Med Rehabil 2012;91:561-9.

37. López-Rodríguez MM, Castro-Sánchez AM, FernándezMartínez M, Matarán-Peñarrocha GA, Rodríguez-Ferrer ME. Comparison between aquatic-biodanza and stretching for improving quality of life and pain in patients with fibromyalgia. Aten Primaria 2012;44:641-9.

38. Esen E, Çetin A. Microvascular functions in patients with fibromyalgia syndrome: effects of physical exercise. Turk J Phys Med Rehab 2017;63:215-23. 